\title{
ANALYSIS OF THE CHARGE TRANSFER IN CCDS INCLUDING THE EFFECT OF SURFACE STATES AND A VARYING FRINGING FIELD
}

\author{
UMESH KUMAR \\ Senior Member, IEEE: Fellow, IETE. Department of Electrical Engg., \\ IIT New Delhi Pin-110016 India. \\ P. BHUSHAN MITAL \\ Fellow, IETE. Department of Electronics \& Communication Engg. \\ C.R. State College of Engineering, Murthal (Sonepat) India Pin-131039.
}

In this paper, a general charge transfer equation is derived that includes the effect of the surface states and does not assume a constant fringing field. Further, a detailed and simple method is presented for the solution of this general charge transfer equation.

\section{INTRODUCTION}

The free charge transfer phenomenon in CCDs has been dealt with in the past by many authors [1, 2, 3, 4]. Most of the authors have, until now, considered a constant fringing field for their analysis. The solution of the charge transfer equation has been obtained [3,4] in the past with the aid of a computer and includes the drift due to the self-induced field and thermal diffusion. The charge transfer phenomenon described in $[1,2,3,4]$ has been concerned with the free electrons in the conduction band only. Nothing has been said about transitions of electrons between the conduction band and surface states.

We present a general charge transfer mechanism here that includes the effect of surface states and assumes a varying fringing field.

\section{SURFACE STATES AND ITS EFFECT ON CHARGE TRANSFER EQUATION}

Let us consider a 3-phase CCD as shown in Fig. 1. In an operating surface channel charge coupled device with no generation current and in the absence of signal charge injection over a long period, the interface states become deeply depleted. If a charge packet is transferred along the device, some fraction of the charge will be trapped in the interface states under each gate. If the remission time constant is short compared to the time allowed for the transfer, or longer, some carriers will,

Assistant Professor, Department of Electrical Engineering, I.I.T., Hauz Khas, New Delhi-110016, India Phone: + (91) - (11) - 3263535 (Residence) + (91) - (11) - 666979/Extn. 2218 (Office) FAX: + (91) $-(11)-6862037$ (Office) 


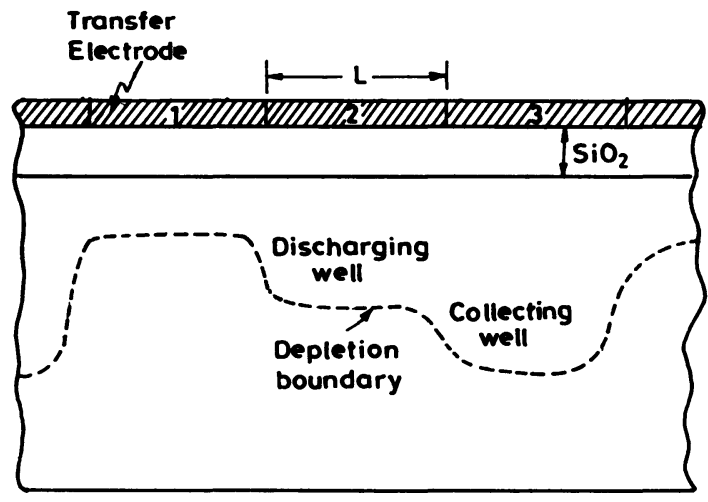

FIGURE 1

in part, join the main packet, while others will be re-emitted only much later and, thus, will form a residual in the following packets. But even if the charge trapped in interface states is re-emitted into the main packet, this is a much slower process than the free charge transfer. Thus, after sometime from the beginning of the cycle, transfer of the charge becomes limited by the rate of emission from the interface states.

Consider a $p$ channel device shown in Fig. 1. The equation describing the occupation of the interface states at the Energy level $E$ above the valence band is given by

$\frac{d n_{s s}}{d t}=K_{1}\left(N_{s s}-n_{s s}\right) p-K_{2} n_{s s} \exp (-E / K T)$

where

$n_{s s}=$ Density of filled states

$N_{s s}=$ Interface state density

$p=$ density/unit area of mobile holes.

and

$K_{1}=\sigma_{h} \nu_{t h} / d$

$K_{2}=\sigma_{h} \nu_{t h} N_{v}$

where

$\sigma_{h}=$ Trap cross section of holes

$\nu_{t h}=$ Average thermal velocity of mobile carriers.

$N_{v}=$ Density of states in the valence bands

$d=$ Average thickness of inversion layer. 
Thus, we can say that the first term in eq. (1) describes the rate of capture of mobile carriers and the second term describes the rate of emission of the trapped carriers. The total rate of emission from the interface states is then given by

$\alpha(x, t)=-\frac{\partial p_{t r}}{\partial t}=-\frac{\partial}{\partial t} \int_{0}^{E_{g}} n_{s s} d E$

where

$E_{g}=$ Energy gap

$p_{t r}=$ Total number of trapped carriers

Thus, the mobile carrier continuity equation must be modified to include this emission rate.

$\frac{\partial p}{\partial t}=-\frac{1(\partial J)}{q(\partial x)}+\alpha(x, t)$

Here

$J=q\left[\mu E_{f} p-\left(\frac{q \mu p}{C_{0 x}}+D\right) \frac{\partial p}{\partial x}\right]$

Thus,

$\frac{\partial p}{\partial t}=\frac{\partial}{\partial x}\left[\left(\frac{q \mu p}{C_{0 x}}+D\right) \frac{\partial p}{\partial x}-\mu E_{f} p\right]+\alpha(x, t)$

Equation (4) is the general equation that relates time and space dependence of the carrier concentration. The boundary conditions and initial values are:

$$
\begin{aligned}
J(0, t) & =0 \\
p(L, t) & =0 \\
\text { and } p(x, 0) & =P_{0} \text { for } 0 \leq x<L
\end{aligned}
$$

Before going on to the solution of this equation, let us first consider the procedure used to determine $p(x, t)$. We assume, according to the charge control model, an expression of the form

$p(x, t)=\phi(x) \exp \left(-t / \tau_{f}\right)$

where

$\phi(x)=$ some function describing the $x$ dependence of $p(x, t)$ for all $t$.

$\tau_{f}=$ decay time constant. 
Now, substituting equation (5) into equation (1) we get a solution for $n_{s s}$. If $K_{1} \tau_{f} p(x, t)>1$, then

$n_{s s}(x, t, E)=N_{s s}\left[1-\frac{K_{2} \exp (-E / K T)}{K_{1} p(x, t)}\right]^{-1}$

Thus, the interface states have a small effective time constant and can equilibrate very rapidly with the mobile carriers. Assuming a constant interface state density $N_{s s}$ and a constant cross section area $\sigma_{h}$ and if

$\frac{1}{K_{2}}<\frac{1}{K, p(x, t)}<\frac{1}{K_{2}} \exp \left(E_{g / k T}\right)$

then the total density of trapped carriers reduces to

$p_{t r(x, t)}=N_{s s}\left[E_{g}-K T \ln \left(\frac{K_{2}}{K_{1} p(x ; t)}\right)\right]$

and using eqn. (2)

$\alpha=\frac{N_{s s} K T}{\tau_{f}}$

Now, if the mobile carrier density falls to a level such that $K_{1} \tau_{f} p(x, t)<1$, the mobile charge is no longer in equilibrium with the trapped charge, which means that the filling time constant of the interface states becomes greater than the time constant governing the variation of mobile carrier density and hence the trap occupation fails to follow the variation of the free charge. In this case, if

$t>t_{1}+\frac{1}{K, p(x, t)}$

where $t_{1}$ is the time, emission of carriers becomes dominant and refilling of the traps becomes negligible given by the equation

$K_{1} \tau_{f} p(x, t)=1$

then

$p_{t r}(x, t)=N_{s s}\left[E_{g}-K T \ln K_{2}\left(t-t_{1}\right)-\frac{K T}{\left(t-t_{1}\right) K_{1}} p\left(x, t_{1}\right)\right]$

and the emission rate is

$\alpha(x, t)=N_{s s} K T\left[\frac{1}{\left(t-t_{1}\right)}-\frac{\tau_{f}}{\left(t-t_{1}\right)^{2}}\right]$ 
Note that inequality (9), which reads $t>t_{1}+\tau_{f}$, means that $\alpha$ cannot be $>-v e$. But more than that, after $t>t_{1}, \alpha$ has no physical reason to increase. It follows that solution (12) is valid only after $\alpha$ has reached its maximum value, which happens at $t=t_{1}+2 \tau_{f}$. Thus, for $t_{1}<t<t_{1}+2 \tau_{f}$ the solution will be

$\alpha \cdot(x, t)=\frac{N_{s s} K T}{\tau_{f}}\left(1-\frac{t_{1}}{\tau_{f}}\right)$

\section{SOLUTION OF THE CHARGE TRANSFER EQUATION}

The charge transfer equation given by eq. (8) by

$\frac{\partial p}{\partial t}=\frac{\partial}{\partial x}\left[\left(\frac{q \mu p}{C_{0 x}}+D\right) \frac{\partial p}{\partial x}-\mu E_{f} p\right]+\alpha(x, t)$

can be written as

$\frac{\partial p}{\partial t}=\frac{\partial H}{\partial x}+\alpha(x, t)$

where

$H=-\frac{1}{q} J=\left(\frac{q \mu p}{C_{0 x}}+D\right) \frac{\partial p}{\partial x}-\mu E_{f} p$

and thus the boundary condition becomes

$H(0, t)=0 \quad(t>0)$

Considering the Linearized Crank Nicholson scheme, if the derivative of $H$ at a net point $j(j=1,2,3 \ldots N-1)$ is expanded, equation (15) can be expressed as given below. Also, by replacing the value of $\alpha(x, t)$ as given by eq. (8)

$\frac{p_{j, k+1}-p_{j, k}}{\tau}=\frac{1}{h}\left(H_{j+1 / 2, k+1 / 2}-H_{j-1 / 2, k+1 / 2}\right)$

with

$$
\begin{aligned}
H_{j \pm 1 / 2, k+1 / 2} & =\frac{1}{2}\left\{\left[\left(\beta \mu p_{j \pm 1 / 2, k}+D\right)\left(\frac{\partial p}{\partial x}\right)_{j \pm 1 / 2, k+1}-\mu E_{f_{(j \pm 1 / 2)}} p_{j \pm 1 / 2, k}\right]\right. \\
& \left.+\left[\left(\beta \mu p_{j \pm 1 / 2, k+1}+D\right)\left(\frac{\partial p}{\partial x}\right)_{j \pm 1 / 2, k}-\mu E_{f_{(j \pm 1 / 2)}} p_{j \pm 1 / 2, k+1}\right]\right\}
\end{aligned}
$$


where

$$
\begin{aligned}
\beta=\frac{q}{C_{0 x}} & \\
\left(\frac{\partial p}{\partial x}\right)_{j+1 / 2, m} & =\frac{p_{j+1, m}-p_{j, m}}{h} \\
\left(\frac{\partial p}{\partial x}\right)_{j-1 / 2, m} & =\frac{p_{j, m}-p_{j-1, m}}{h} \\
p_{j \pm 1 / 2, m} & =\frac{p_{j \pm 1, m}+p_{j, m}}{2} \quad\left\{\begin{array}{c}
m=k \\
\text { and } \\
m=k+1
\end{array}\right\} \\
h & =\frac{1}{N-1}
\end{aligned}
$$

After some algebraic manipulations, equation (17) becomes

$A_{j} p_{j+1, k+1}+B_{j} p_{j, k+1}+C_{j} p_{j-1, k+1}=R_{j}$

$J=2,3,4 \ldots N-1$

where

$$
\begin{aligned}
& A_{j}=\frac{-B \mu \tau}{2 h^{2}} p_{j+1, k}-\frac{D \tau}{2 h^{2}}+\frac{\mu \tau}{4 h} E_{f_{(j+1 / 2)}} \\
& B_{j}=1+\frac{\beta \mu \tau}{2 h^{2}} p_{j, k}+\frac{D \tau}{2 h^{2}}+\frac{\mu \tau}{4 h}\left[E_{f_{(j+1 / 2)}}-E_{f_{(j-1 / 2)}}\right] \\
& C_{j}=\frac{-\beta \mu \tau}{2 h^{2}} p_{j-1, k}-\frac{D \tau}{2 h^{2}}-\frac{\mu \tau}{4 h} E_{f_{(j-1 / 2)}}
\end{aligned}
$$

and

$$
\begin{aligned}
R_{j}= & p_{j, k}+\frac{D \tau}{2 h^{2}}\left(p_{j+1, k}-2 p_{j, k}+p_{j-1, k}\right) \\
& -\frac{\mu \tau}{4 h}\left[E_{f_{(j+1 / 2)}}\left(p_{j, k}+p_{j+1, k}\right)-E_{f_{(j-1 / 2)}}\left(p_{j, k}+p_{j-1, k}\right)\right] \\
& -\frac{N_{s s} k T}{\tau_{f}}
\end{aligned}
$$


For $j=1$, using

$p_{1, k+1}-p_{1, k}=\frac{1}{h} H_{1 / 2, k+1}$

with equation (18) and (19), we get

$A_{1} p_{2, k+1}+B_{1} p_{1, k+1}=R_{1}$

where

$$
\begin{array}{ll}
A_{1}= & \frac{-\beta \mu \tau}{2 h^{2}} p_{2, k}-\frac{D \tau}{2 h^{2}}+\frac{\mu \tau}{4 h} E_{f_{(3 / 2)}} \\
B_{1}= & 1+\frac{\beta \mu \tau}{2 h^{2}} p_{1, k}+\frac{D \tau}{2 h^{2}}+\frac{\mu \tau}{4 h} E_{f_{(3 / 2)}} \\
\text { and } & \begin{array}{ll}
R_{1}=\quad & p_{1, k}+\frac{D \tau}{2 h^{2}}\left(p_{2, k}-2 p_{1, k}+p_{0, k}\right) \\
& -\frac{\mu \tau}{4 h} E_{f_{(3 / 2)}}\left[p_{2, k}+p_{1, k}\right]-\frac{N_{s s} k T}{\tau_{f}} \\
& \because E_{f_{(1 / 2)}}=0
\end{array}
\end{array}
$$

and for $j=N$

$p_{N, k+1}=0$

Now, equations $(20,(23)$ and (25) become a system of $N$-linear algebraic equations with a tridiagonal coefficient matrix. This can now be solved by any of several methods such as Gauss-elimination, etc. Note that here two time levels have been considered, namely $(K+1) \tau$ and $K \tau$. Time derivative is taken as the backward difference at point $(j, k+1)$ and the space derivatives are expressed by the values at $(K+1) \tau$.

Since it is obvious that all the unknowns are in $(K H)$, the second subscripts can be omitted for simplicity. Let

$p_{j}=G_{j} p_{j+1}+F_{j}$ for $j=1,2,3 \ldots N-1$

then

$p_{j-1}=G_{j-1} p_{j}+F_{j-1}$ for $j=2,3,4 \ldots N$ 
Substituting (27) into (20) and rearranging terms we get

$p_{j}=-\frac{A_{j}}{B_{j}+C_{j} G_{j-1}} p_{j+1}+\frac{R_{j}-C_{j} F_{j-1}}{B_{j}+C_{j} G_{j-1}}$ for $j=2,3,4 \ldots N-1$

Comparing (28) with (26) we have

$$
\begin{aligned}
G_{j} & =-\frac{A_{j}}{B_{j}+C_{j} G_{j-1}} \text { for } j=2,3,4 \ldots N-1 \\
F_{j} & =\frac{R_{j}-C_{j} F_{j-1}}{B_{j}+C_{j} G_{j-1}} \quad \text {-do- }
\end{aligned}
$$

Also comparing (23) with (26).

$G_{1}=-A_{1} / B_{1}$ and $F_{1}=R_{1} / B_{1}$

Since $p_{N}$ is given by eq. 25 , back substitution of $j=N-1, N-2 \ldots 2,1$ in (26) with all the $G$ 's and $F$ 's, all the $p_{j}$ can be determined.

\section{CONCLUSIONS}

This paper presents the charge transfer phenomenon in CCDs taking into consideration two factors-varying fringing field and surface states. A general equation has been formulated including these effects, and a method has been suggested for the solution of this general equation.

\section{REFERENCES}

1. S. M. Sze, Physics of Semiconductor Devices, second edition, John Wiley and sons, New York-1981.

2. Charge Transfer Devices: Sequin \& Tompsel, Academic Press, Inc..

3. C. K. Kim and M. Lenzlinger, "Charge Transfer in CCD," Journal of Applied Physics, Vol. 42, p. 3586, Aug. 70.

4. R. J. Strain and N. L. Schryer, "A Non-linear Diffusion Analysis of Charge transfer," Bell System Technical Journal, Vol. 50, p. 1721, July-1971. 

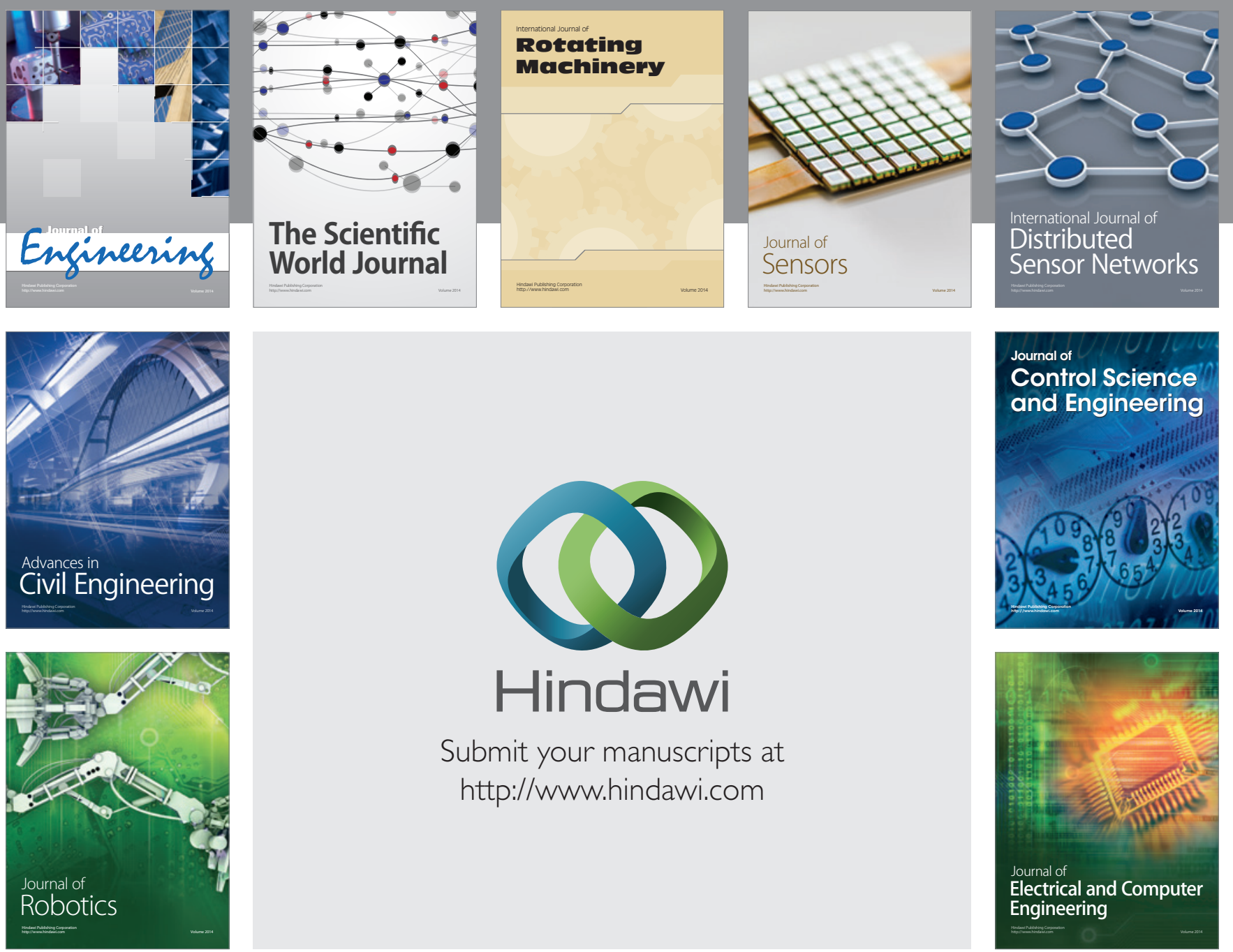

Submit your manuscripts at

http://www.hindawi.com
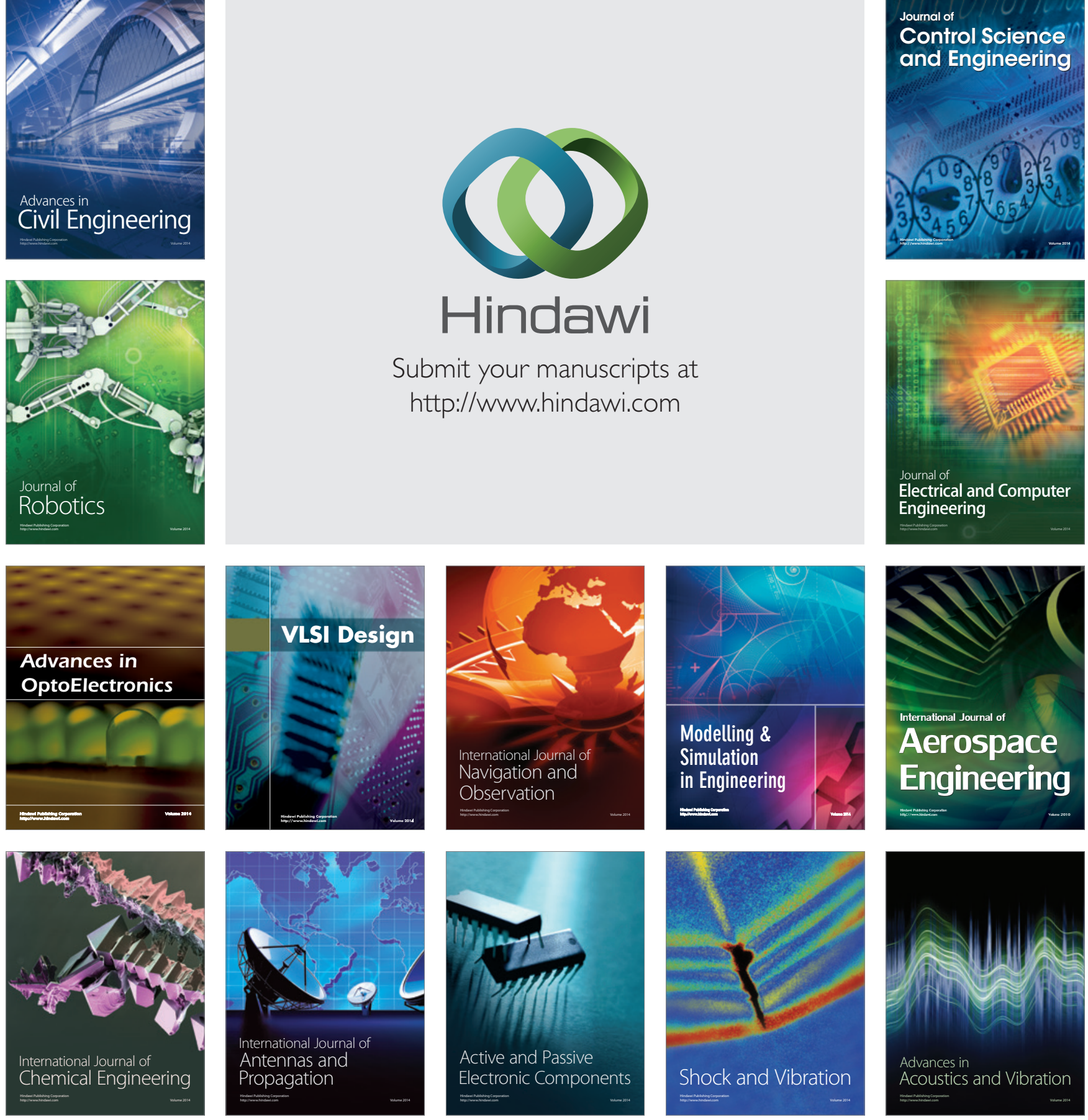